\title{
Densidad, biomasa y composición del zooplancton, en el estrato superficial de la cuenca de Cariaco, Venezuela
}

\author{
Density, biomass and composition of zooplankton, in the surface layer \\ of the Cariaco basin, Venezuela \\ Brightdoom Márquez ${ }^{1}$, José Díaz-Ramos ${ }^{1}$, Luis Troccoli², \\ Baumar Marín ${ }^{1}$ y Ramón Varela ${ }^{3}$ \\ ${ }^{1}$ Laboratorio de Zooplancton, Departamento de Biología Marina, Instituto Oceanográfico de Venezuela, \\ Universidad de Oriente. Apdo. Postal 245, Cumaná, Sucre 6101, Venezuela \\ ${ }^{2}$ Instituto de Investigaciones Científicas, Universidad de Oriente, Núcleo de Nueva Esparta. \\ ${ }^{3}$ EDIMAR, Fundación de Ciencias Naturales La Salle, Campus de Nueva Esparta, \\ Pta. de Piedras, Nueva Esparta, Venezuela \\ bmarquez@sucre.udo.edu.ve
}

\begin{abstract}
In order to understand the processes that determine changes in zooplankton abundance and composition in the CARIACO (Carbon Retention in a Colored Ocean) Time Series Station, zooplankton biomass, composition and density were studied aboard the O/V Hermano Ginés from November 2002 to January 2003. Salinity, temperature and dissolved oxygen were measured in situ. Zooplankton samples were collected by vertical tows using a Bongo net (mesh size 333 and $555 \mathrm{~mm}$ ) at a speed of 2 knots at the intervals 0-30, 30-70, and 70-100 m. Chlorophyll (chl $a$ ), zooplankton biomass (dry mass) and density were estimated. The maximum values of biomass $\left(0.039 \mathrm{mg} \mathrm{m}^{-3}\right.$ dry mass) and zooplankton density (582 ind. $\mathrm{m}^{-3}$ ) for the three strata were measured during the upwelling period. On the other hand, the lower values for these variables $\left(0.030 \mathrm{~mL} \mathrm{~m}^{-3}, 0.007 \mathrm{mg} \mathrm{m}^{-3}\right.$ and 7 ind. $\mathrm{m}^{-3}$, respectively) were recorded in May 2003. In general, the greatest values were detected within the 30 to $70 \mathrm{~m}$ range. Even though, the variability of the different strata was similar. The zooplankton community was dominated by copepods, cladocerans and appendicularians. The results showed a great temporal variability en zooplankton biomass and composition in spite of the fact that the station is located in a tropical area due to the effect of seasonal coastal upwelling.
\end{abstract}

Key words: Plankton, coastal upwelling, Caribbean Sea
Resumen.- Con la finalidad de entender los procesos que determinan los cambios en la composición y abundancia del zooplancton en la estación de series de tiempo CARIACO ('Carbon Retention in a Colored Ocean'), se estudió la densidad, biomasa y composición del zooplancton durante el periodo noviembre 2002-enero 2004. Se midió in situ la salinidad, temperatura y concentración de oxígeno disuelto. Se estimó la biomasa fitoplanctónica ( $\mathrm{Chl} a$ ), la biomasa (peso seco) y densidad zooplanctónica. Se realizaron calados oblicuos bimensuales con una red Bongo en los intervalos 0-30, 30-70 y 70-100 m. Los máximos valores de biomasa $\left(0,039 \mathrm{mg} \mathrm{m}^{-3}\right.$ peso seco) y densidad zooplanctónica (582 ind. $\mathrm{m}^{-3}$ ) para las tres capas se midieron en el período de surgencia costera. Por otra parte, los valores más bajos para esta variables $(0,007 \mathrm{mg}$ $\mathrm{m}^{-3}$ and 7 ind. $\mathrm{m}^{-3}$, respectivamente) se registraron en mayo de 2003. En general, los mayores valores se detectaron en la capa 30-70 m, a pesar de que la variabilidad temporal en los estratos fue similar. La comunidad zooplanctónica estuvo dominada por copépodos, cladóceros y apendicularias. Los resultados reflejaron una amplia variabilidad temporal en la composición y abundancia de la comunidad zooplanctónica, a pesar de que la estación está ubicada en un área tropical, debido al efecto de la surgencia costera estacional.

Palabras clave: Plancton, surgencia costera, Mar Caribe

\section{Introducción}

En el medio nerítico, los patrones de distribución espacial comunitaria y la diversidad son el resultado de la evolución, la biogeografía y más aún, el resultado de factores abióticos y bióticos locales que operan a diferentes escalas temporales y espaciales (Angel 1998, Berasategui 2003).

La surgencia costera constituye uno de los factores determinantes sobre estos patrones de distribución, como ocurre en la zona nororiental de Venezuela incluyendo la cuenca de Cariaco (Fukuoka 1965, Astor et al. 1998). Este fenómeno ocurre durante el período de menor pluviosidad cuando la profundidad de la capa de mezcla aumenta, en contraste con el periodo de lluvias y vientos de menor velocidad en el país cuando la columna de agua se estratifica (Okuda \& Benítez 1974). Se ha determinado que este proceso no solo depende del aumento de la intensidad del viento, sino de otros factores como giros ciclónicos, zonas de divergencia, ondas internas atrapadas 
en la plataforma, o intensificación del giro anticiclónico del Océano Atlántico (Gómez 1996, Müller-Karger et al. 1999).

La cuenca de Cariaco es un cuerpo de agua particularmente importante desde el punto de vista científico. Además de su profundidad $(1.390 \mathrm{~m})$ y de su ubicación dentro de la plataforma continental venezolana, sus aguas son anóxicas de forma permanente por debajo de los $300 \mathrm{~m}$, lo cual es un fenómeno que también se produce también en el mar Negro (Van Geen et al. 1995).

La cuenca de Cariaco ha sido objeto de diversos estudios, en su mayor parte relacionados con características hidrográficas y aspectos fisicoquímicos (Richards \& Vaccaro 1956, Richards 1960, Okuda et al. 1969, Febres 1974, Walsh et al. 1999, Scranton et al. 2001, Müller-Karger et al. 2001). El zooplancton de la cuenca de Cariaco ha recibido poca atención y los estudios existentes se remontan a los años 60, cuando Zoppi (1961) determinó la biomasa de estos organismos. Legaré (1961) identificó 64 especies de copépodos mientras que Cervigón \& Marcano (1965) analizaron la abundancia y distribución de los copépodos desde los $150 \mathrm{~m}$ hasta la superficie. Urosa (1983) estudio la distribución del zooplancton en la cuenca Tuy-Cariaco, observando que los mayores valores de biomasa y densidad se obtuvieron en los primeros meses del año, como consecuencia del fenómeno de surgencia. Más recientemente, Peñuela (2000) estableció que no existían diferencias significativas en la biomasa entre biomasa zooplanctónica diurna y nocturna.

En el presente estudio se relaciona la densidad, biomasa y composición del zooplancton con las condiciones hidrográficas en la estación de series de tiempo CARIACO (Carbon Retention in a Colored Ocean), a fin de establecer los posibles patrones de sedimentación/exportación de la materia orgánica de origen zooplanctónica hacia la zona anóxica de la cuenca de Cariaco.

\section{Material y métodos}

Se realizaron muestreos bimensuales en el estrato 0-100 $m$ en la estación de series de tiempo CARIACO (ubicada a $10^{\circ} 30^{\prime} \mathrm{N}$ y $64^{\circ} 40^{\prime} \mathrm{O}$ ) con una profundidad de $1.300 \mathrm{~m}$ a bordo de B/O HERMANO GINÉS de la Estación de Investigaciones Marinas (EDIMAR) de la Fundación La Salle (FLASA) durante el periodo noviembre 2002-enero 2004. La salinidad, temperatura, concentración de oxígeno disuelto y concentración de clorofila $a(\mathrm{Chl} a)$ se midieron a partir de las muestras de agua con botellas de Niskin (5 L) adosadas a una roseta a $0,7,15,25,35$, 55,75 y $100 \mathrm{~m}$. La salinidad y la temperatura se midieron por conductividad con un salinómetro automatizado Autosal Guildline ${ }^{\circledR}$ y con un termómetro de inmersión SBE 25 CTD Seabird ${ }^{\circledR}$, respectivamente. La concentración de oxígeno disuelto se determinó por el método de titulación modificado de Winkler (Strickland \& Parsons 1972). Por otra parte, la concentración de fosfato, nitrato y de Chl $a$ se establecieron de acuerdo a las recomendaciones de Gordon et al. (1993) y de Parsons et al. (1984), respectivamente.

Las muestras para estudiar la biomasa y composición del zooplancton se recolectaron con una red tipo Bongo de $60 \mathrm{~cm}$ de diámetro de boca, equipada con mangas de $333 \mathrm{~mm}$ y $555 \mathrm{~mm}$ de abertura de malla a las cuales se les colocó un medidor de flujo a fin de cuantificar el volumen de agua filtrada. Los calados se realizaron a tres profundidades $(0-30 ; 30-70$ y 70-100 m) con una duración de $15 \mathrm{~min}$, a una velocidad de arrastre de 2 nudos.

Las muestras se fijaron con formaldehído al $4 \%$, neutralizado con borato de sodio. Cada muestra se homogenizó y se dividió en dos porciones iguales por medio de un separador de Folsom (McEven et al. 1954); una porción fue destinada a estudios de estructura de la comunidad y la otra para el análisis de la biomasa por medio del método gravimétrico (peso seco, Postel et al. 2000). Para la ubicación taxonómica de los organismos se tomaron tres alícuotas de $2,5 \mathrm{~mL}$ y se procedió al conteo e identificación utilizando los trabajos de Tregouboff \& Rose (1957), Smith (1977), Boltovskoy (1981), Gasca \& Suárez (1996), Boltovskoy (1999) y Martin \&Davis (2001).

Las pruebas de normalidad (Shapiro Wilks) y de homogeneidad de varianza (Bartlet) determinaron la aplicación del análisis no paramétrico Kruskal-Wallis (Siegel \& Castellan 1995) para evaluar si las variables bióticas y abióticas fueron significativamente diferentes entre los estratos estudiados (profundidad de calado) y de la prueba Mann-Whitney en cuanto a temporadas (surgencia y relajación) (Fukuoka 1965, Okuda \& Benítez 1974). Todos los resultados se expresaron en diagramas de caja y bigotes de acuerdo a la recomendación de Boyer et al. (1997).

Para establecer las relaciones entre las variables estudiadas y los grupos zooplanctónicos se realizó un análisis de correspondencia canónica (Johnson \& Wichern 1992) y para establecer la relación entre los diferentes grupos de organismos se realizó un análisis de clasificación ('cluster') utilizando el índice de Bray Curtis con el algoritmo de la distancia euclidiana (Legendre \& Legendre 1998). 


\section{Resultados}

\section{Variables abióticas}

Los estadísticos básicos de las variables estudiadas se presentan en la Tabla 1. En general, el estrato superficial (0-30 m) presentó la temperatura del agua más cálida $\left(21,9-28,5^{\circ} \mathrm{C}\right)$, en el estrato intermedio la temperatura fluctuó entre $21,4-25,8^{\circ} \mathrm{C}$, mientras que en el último las temperaturas fueron más bajas $\left(<20,9^{\circ} \mathrm{C}\right)$, detectándose diferencias significativas entre los estratos (KruskalWallis Kw =7,64; $P<0,05$; Fig. 1A). Con respecto a los meses estudiados, durante el período de surgencia, entre enero y julio de 2003 , los valores fueron bajos y fluctuaron entre $19,7-23,1^{\circ} \mathrm{C}$ (Fig. 1B), mientras que para el período de relajación (noviembre 2002, septiembre y noviembre 2004$)$ fueron más cálidos $\left(23,1-28,5^{\circ} \mathrm{C}\right)$. Por otra parte, se demostró la existencia de cambios significativos en la temperatura del agua entre los dos períodos $(\mathrm{Kw}=116 ; P<0,05)$. A diferencia de la temperatura, la salinidad no mostró diferencias significativas con respecto a los estratos $(\mathrm{KW}=7,49 ; P$ $>0,05)$. En general, el estrato superior presentó los valores más elevados (>36,5) (Fig. 1C); sin embargo, durante los meses correspondientes al período de relajación, el gradiente vertical fue inverso, es decir, las salinidades más bajas se midieron en la capa superficial (Fig. 1D). Durante el período de surgencia la salinidad alcanzó valores $>36,8$; no obstante, a pesar de la diferencias observadas en la salinidad durante las épocas, no se detectaron diferencias significativas $(\mathrm{Kw}=0,11$; $P>0,05$; Fig. 1D).
El oxígeno disuelto también presentó diferencias significativas por estrato $(\mathrm{Kw}=15,41 ; P<0,05$; Fig. 1E) con la mayor concentración $\left(>4,0 \mathrm{~mL} \mathrm{~L}^{-1}\right)$ en el estrato superficial. Sin embargo, no mostró diferencias significativas para las épocas $(\mathrm{Kw}=15,45 ; P<0,05)$ (Fig. 1F); durante el período de surgencia las concentraciones fluctuaron entre $2,95-5,54 \mathrm{~mL} \mathrm{~L}^{-1}$.

El nitrato (NO) varió significativamente por estrato $(\mathrm{Kw}=15,31 ; P<0,05)$ registrándose las mayores concentraciones ( $>4 \mathrm{mg} \mathrm{m}^{-3}$; Fig. 2A) en el estrato más profundo del estudio (70-100 m). Al igual que el oxígeno, en el nitrato no se registraron diferencias entre las épocas $(\mathrm{Kw}=43 ; P>0,05 ;$ Fig. 2B). El fosfato (PO) siguió el mismo comportamiento que el nitrato, los valores más altos se detectaron en el estrato profundo (>3 $\mathrm{mg} \mathrm{m}^{-3}$; Fig. 2C), variando significativamente con respecto a los estratos (Kw $=14,94 ; P<0,05$; Fig. 2C); no obstante, entre las épocas las concentraciones fueron muy similares, no registrándose diferencias $(\mathrm{Kw}=43 ; P>0,05$; Fig. 2D).

\section{Variables bióticas}

El análisis del comportamiento vertical de la clorofila $a$ reveló diferencias significativas $(\mathrm{Kw}=10,52 ; P<0,05)$ entre la superficie y el resto de la columna de agua (Fig. $2 \mathrm{E})$. La clorofila $a$ no varió significativamente entre las dos épocas, aunque en enero y marzo del 2003 se midieron valores mayores que $2,5 \mathrm{mg} \mathrm{m}^{-3}(\mathrm{Kw}=42,5 ; P>0,05$; Fig. 2F).

\section{Tabla 1}

Estadísticos básicos de las variables abióticas y bióticas medidos en el estrato superficial de la Cuenca de Cariaco, Estado Sucre, Venezuela

Basic statistics of the biotic and abiotic measured variables in the surface layer of the Cariaco basin, Sucre state, Venezuela

\begin{tabular}{|c|c|c|c|c|}
\hline Variables & Promedio & D.E. & Mínimo & Máximo \\
\hline Salinidad & 36,84 & 0,107 & 36,59 & 36,99 \\
\hline Temperatura $\left({ }^{\circ} \mathrm{C}\right)$ & 23,11 & 2,576 & 18,61 & 28,47 \\
\hline Oxígeno disuelto $\left(\mathrm{mL} \mathrm{L}^{-1}\right)$ & 3,87 & 0,890 & 2,11 & 5,54 \\
\hline Fosfato $\left(\mathrm{mg} \mathrm{m}^{-3}\right)$ & 1,86 & 1,48 & 0,06 & 4,72 \\
\hline Nitrato $\left(\mathrm{mg} \mathrm{m}^{-3}\right)$ & 2,99 & 2,86 & 0,01 & 7,33 \\
\hline Biomasa fitoplanctónica (mg Chl $a \mathrm{~m}^{-3}$ ) & 0,48 & 0,914 & 0,045 & 3,09 \\
\hline Volumen desplazado $\left(\mathrm{mL} \mathrm{m}^{-3}\right)$ & 0,091 & 0,098 & 0,007 & 0,354 \\
\hline Peso seco $\left(\mathrm{mg} \mathrm{m}^{-3}\right)$ & 0,014 & 0,010 & 0,001 & 0,039 \\
\hline
\end{tabular}



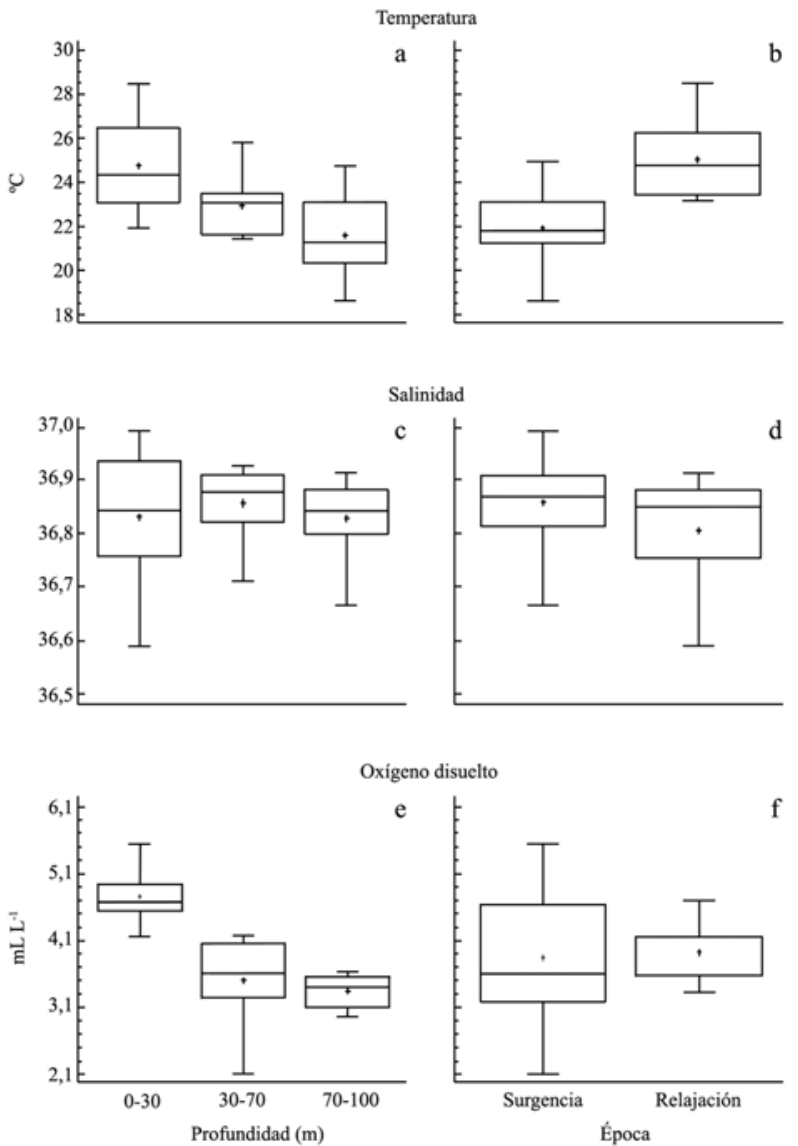

Figura 1

Variación por estrato y por época de la temperatura (A y

B), salinidad (C y D) y del oxígeno disuelto (E y F) del estrato superficial de la cuenca de Cariaco, Estado Sucre, Venezuela

Variation for stratum and epoch of the temperature (A and $\mathrm{B})$, salinity (C and $\mathrm{D})$, and dissolved oxygen ( $\mathrm{E}$ and $\mathrm{F}$ ) from surface layer of the Cariaco Basin,

Sucre state, Venezuela

Los valores de biomasa zooplanctónica (peso seco) oscilaron entre 0,001 y $0,039 \mathrm{mg} \mathrm{m}^{-3}$ (Fig. 3A). Durante el período comprendido entre enero y marzo 2003 (época de surgencia) se registraron valores relativamente altos de peso seco $\left(0,020 \mathrm{mg} \mathrm{m}^{-3}\right.$, para ambos meses) en los primeros metros de la columna de agua $(<20 \mathrm{~m})$; sin embargo, en marzo también se detectaron valores altos en la zona profunda (> $\left.80 \mathrm{~m} ; 0,024 \mathrm{mg} \mathrm{m}^{-3}\right)$. En el período mayo-julio 2003 se midieron los valores más bajos a todo lo largo de la columna de agua, con valores promedios de $0,007 \mathrm{mg} \mathrm{m}^{-3}$; posteriormente en noviembre se
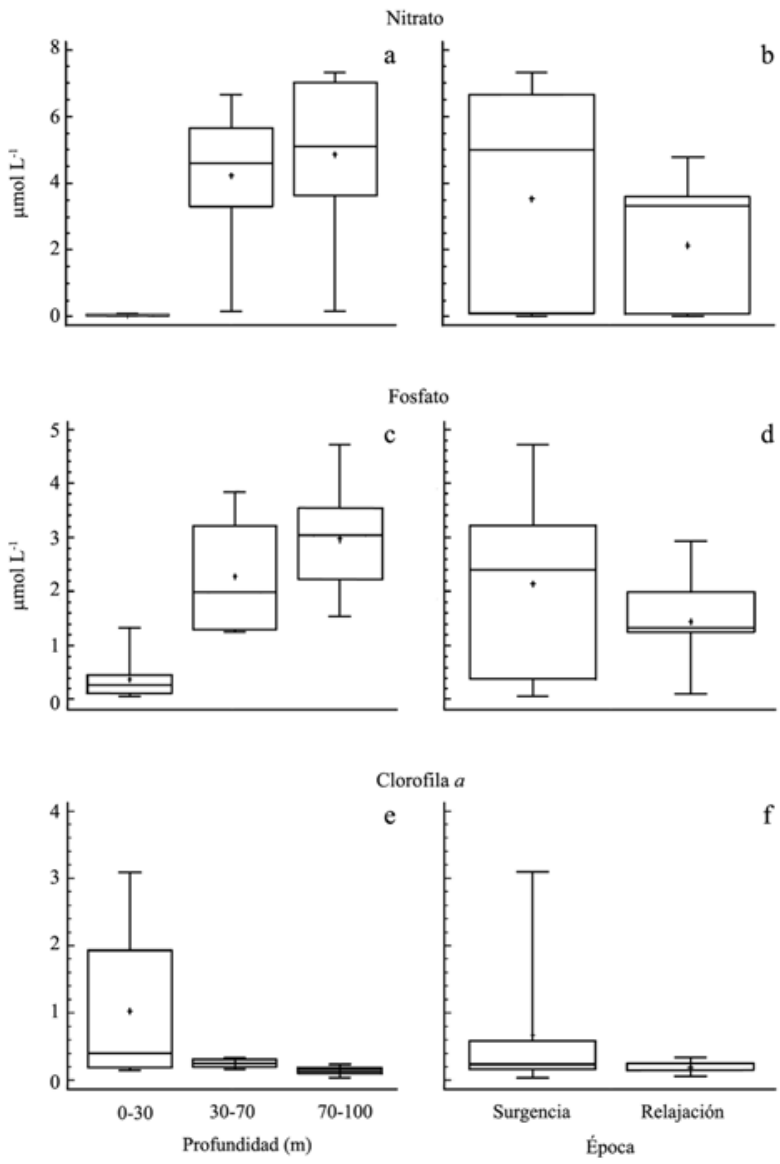

Figura 2

Variación por estrato y por época del nitrato (A y B), fosfato (C y D) y de la clorofila $a(E$ y $F)$ del estrato superficial de la cuenca de Cariaco, Estado Sucre, Venezuela

Variation for stratum and epoch of the nitrate (A and B), phosphate (C and D) and chlorophyll $a(\mathrm{E}$ and $\mathrm{F})$ from surface layer of the Cariaco Basin,

Sucre state, Venezuela

registraron los valores más altos del estudio, entre los $70-100 \mathrm{~m}$ de profundidad $\left(0,039 \mathrm{mg} \mathrm{m}^{-3}\right.$ de peso seco) (Fig. 3A).

Se detectaron diferencias significativas en el peso seco con respecto a los meses $(\mathrm{Kw}=5,09 ; P<0,05)$, mientras que por estrato no se encontraron diferencias significativas.

La distribución vertical de la densidad zooplanctónica (Fig. 3B) durante los ocho meses de estudio, mostró valores entre 7-582 ind. $\mathrm{m}^{-3}$. Las cifras más bajas se obtuvieron en enero 2004 para el nivel más profundo, 


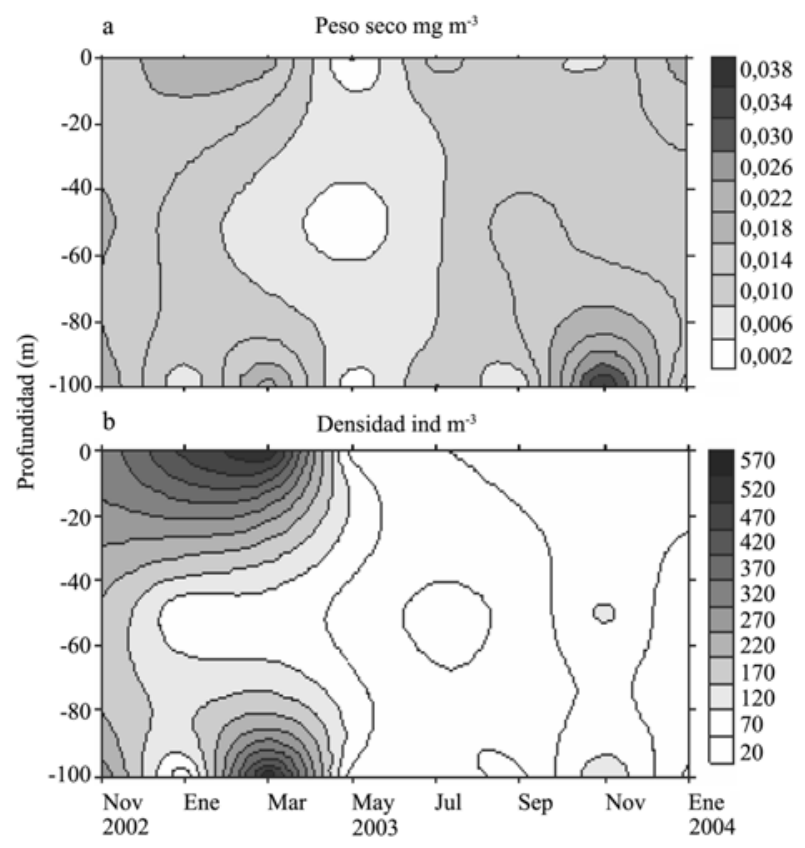

Figura 3

Variación mensual y por estratos de la biomasa zooplanctónica expresada en (A) Peso seco $\left(\mathrm{mg} \mathrm{m}^{-3}\right)$ y (B) Variación mensual y vertical de la densidad zooplanctónica (ind. $\mathrm{m}^{-3}$ ) del estrato superficial de la cuenca de Cariaco, Estado Sucre, Venezuela

Monthly variation and zooplanktonic biomass stratification expressed by (A) dry mass $\left(\mathrm{mg} \mathrm{m}^{-3}\right)$ and (B) monthly variation and vertical zooplanktonic density (ind. $\mathrm{m}^{-3}$ ) from surface layer of the Cariaco Basin, Sucre state, Venezuela mientras que los más altos se registraron en marzo 2003 en toda la columna de agua $(0-100 \mathrm{~m})$. Por estratos, la densidad varió entre 41-571 ind. $\mathrm{m}^{-3}$ para el estrato superficial $(0-30 \mathrm{~m})$, mientras que para la profundidad de 30-70 m las cifras oscilaron entre 4-219 ind. $\mathrm{m}^{-3} \mathrm{y}$ para la capa más profunda estudiada $(70-100 \mathrm{~m})$ variaron entre 7-582 ind. $\mathrm{m}^{-3}$ (Fig. 3B).

En general, se detectaron tres picos de máximos valores de densidad zooplanctónica, en noviembre 2002 y marzo 2003, con los valores más altos durante todo el estudio para los tres estratos, con un promedio de 290 ind. $\mathrm{m}^{-3}$ y 567 ind. $\mathrm{m}^{-3}$, respectivamente; mientras que el tercer pico correspondió a noviembre 2003 con 176 ind. $\mathrm{m}^{-3}$. El período comprendido entre mayo y septiembre 2003 mostró los valores más bajos de densidad zooplanctónica, en los tres estratos estudiados (4-104 ind. $\mathrm{m}^{-3}$ ) (Fig. 2B). La densidad total mostró diferencias significativas entre los meses $(\mathrm{Kw}=15,19 ; P<0,05)$, sin embargo, no se encontraron diferencias entre los estratos estudiados $(\mathrm{Kw}=3,84 ; P>0,05)$.

La fluctuación en ambos holoplancton y meroplancton se ilustran en la Fig. 4. Las densidades totales siempre son más altas para el holoplancton que para las larvas de las formas antes mencionadas (Tabla 2).

\section{Holoplancton}

Dentro del componente holoplancton los grupos más abundantes fueron los copépodos, apendicularias, cladoceros, doliolidos y quetognatos (Tabla 2). Los copépodos resultaron ser el grupo con mayor representación dentro del zooplancton, casi siempre estuvieron por encima del $60 \%$ de la abundancia total en todos los meses estudiados (Tabla 2). Las máximas

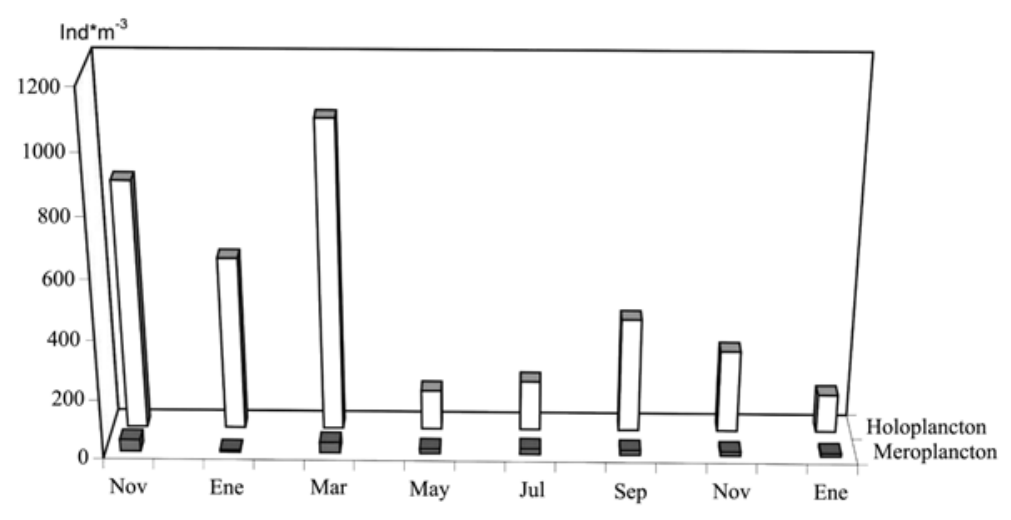

Figura 4

Abundancia total del holoplancton y meroplancton del estrato superficial de la cuenca de Cariaco, Estado Sucre, Venezuela

Total abundance of holo and meroplankton from surface layer of the Cariaco Basin, Sucre state, Venezuela 
Tabla 2

Abundancia mensual (ind. $\mathbf{m}^{-3}$ ) y por estratos del zooplancton de la Cuenca de Cariaco, estado Sucre, Venezuela.

Monthly abundance (ind. $\mathrm{m}^{-3}$ ) and zooplankton stratum of the Cariaco Basin, Sucre State, Venezuela

\begin{tabular}{|c|c|c|c|c|c|c|c|c|c|c|c|c|}
\hline \multirow{2}{*}{$\begin{array}{l}\text { Mes\Año } \\
\text { Estrato(m) }\end{array}$} & \multicolumn{3}{|c|}{ NOVIEMBRE-2002 } & \multicolumn{4}{|c|}{ ENERO-2003 } & \multicolumn{2}{|c|}{ MARZO-2003 } & \multicolumn{3}{|c|}{ MAYO- 2003} \\
\hline & $0-30$ & $30-70$ & $70-100$ & 0 - & & $30-70$ & $70-100$ & $0-30$ & $70-100$ & $0-30$ & $30-70$ & $70-100$ \\
\hline Copépodos & 287 & 156 & 208 & 3 & & 57 & 22 & 401 & 481 & 31 & 34 & 29 \\
\hline Cladóceros & 0 & 1 & 2 & & 3 & 0 & 0 & 31 & 14 & 2 & 2 & 2 \\
\hline Quetognatos & 5 & 1 & 12 & & 0 & 1 & 1 & 14 & 12 & 1 & 1 & 2 \\
\hline Ostracodos & 4 & 1 & 15 & & 5 & 0 & 1 & 5 & 9 & 1 & 2 & 2 \\
\hline Pterópodos & 2 & 2 & 4 & & 5 & 1 & 1 & 2 & 9 & 0 & 0 & 0 \\
\hline Heterópodos & 0 & 0 & 1 & & 0 & 0 & 0 & 0 & 0 & 1 & 0 & 0 \\
\hline Foraminíferos & 5 & 5 & 13 & & 3 & 8 & 3 & 31 & 7 & 3 & 1 & 2 \\
\hline Radiolarios & 0 & 0 & 0 & & 0 & 0 & 0 & 0 & 0 & 0 & 0 & 0 \\
\hline Anfipodos & 0 & 1 & 2 & & 1 & 0 & 1 & 0 & 2 & 0 & 0 & 0 \\
\hline Sifonóforos & 0 & 1 & 1 & & 3 & 1 & 1 & 0 & 0 & 1 & 0 & 2 \\
\hline Medusas & 0 & 0 & 1 & & 1 & 1 & 1 & 0 & 1 & 0 & 0 & 2 \\
\hline Salpidos & 0 & 0 & 0 & & 0 & 0 & 0 & 0 & 0 & 0 & 0 & 0 \\
\hline Apendicularias & 14 & 23 & 22 & & 5 & 2 & 1 & 8 & 0 & 3 & 1 & 2 \\
\hline Doliolidos & 8 & 17 & 12 & & 3 & 1 & 0 & 1 & 0 & 0 & 1 & 2 \\
\hline Eufausidos & 0 & 0 & 0 & & 0 & 0 & 0 & 0 & 0 & 0 & 0 & 0 \\
\hline Balánidos * & 0 & 0 & 0 & & 1 & 0 & 0 & 0 & 8 & 1 & 5 & 1 \\
\hline Bivalvos * & 0 & 0 & 0 & & 0 & 0 & 1 & 0 & 0 & 0 & 0 & 1 \\
\hline Decápodos * & 8 & 8 & 14 & & 1 & 0 & 0 & 6 & 14 & 2 & 2 & 2 \\
\hline Gasterópodos * & 0 & 0 & 1 & & 0 & 0 & 0 & 0 & 0 & 0 & 0 & 0 \\
\hline Ictioplancton * & 2 & 1 & 2 & & 1 & 0 & 0 & 1 & 2 & 0 & 0 & 0 \\
\hline Ictioplancton ** & 0 & 1 & 1 & & 0 & 0 & 0 & 0 & 3 & 1 & 0 & 0 \\
\hline Poliquetos * & 0 & 0 & 1 & & 1 & 0 & 1 & 1 & 1 & 1 & 0 & 0 \\
\hline Larvas Cifonautas & 0 & 1 & 1 & & 1 & 0 & 0 & 0 & 0 & 0 & 0 & 0 \\
\hline Total & 335 & 219 & 313 & & & 72 & 34 & 501 & 563 & 49 & 49 & 50 \\
\hline Mes\Año\} $&{\multicolumn{3}{|c|}{\text { JULIO-2003 }}\text { JULIO-2003 }} &{\multicolumn{3}{|c|}{\text { SEPTIEMBRE-2003 }}\text { SEPTIEMBRE-2003 }} &{\multicolumn{3}{|c|}{\text { NOVIEMBRE-2003 }}\text { NOVIEMBRE-2003 }} &{\multicolumn{3}{|c|}{\text { ENERO-2004 }}\text { ENERO-2004 }} \\
{\hline \text { Estrato(m) }} &{0-30} &{30-70} &{70-100} &{0-30} &{30-70} &{70-100} &{0-30} &{30-70} &{70-100} &{0-30} &{30-70} &{70-100} \\
{\hline \text { Copépodos }} &{114} &{4} &{31} &{84} &{94} &{132} &{172} &{44} &{8} &{46} &{25} &{4} \\
{\hline \text { Cladóceros }} &{1} &{ } &{ } &{7} &{1} &{1} &{1} &{ } &{ } &{15} &{ } &{1} \\
{\hline \text { Quetognatos }} &{1} &{1} &{1} &{1} &{2} &{1} &{5} &{1} &{1} &{ } &{1} &{ } \\
{\hline \text { Ostracodos }} &{2} &{ } &{2} &{ } &{ } &{ } &{1} &{4} &{1} &{ } &{1} &{1} \\
{\hline \text { Pterópodos }} &{ } &{ } &{1} &{ } &{1} &{1} &{1} &{ } &{ } &{1} &{ } &{ } \\
{\hline \text { Heterópodos }} &{0} &{0} &{0} &{0} &{0} &{0} &{0} &{0} &{0} &{0} &{0} &{0} \\
{\hline \text { Foraminíferos }} &{2} &{1} &{ } &{3} &{2} &{3} &{3} &{3} &{1} &{1} &{1} &{ } \\
{\hline \text { Radiolarios }} &{ } &{ } &{ } &{ } &{1} &{2} &{ } &{1} &{ } &{26} &{ } &{ } \\
{\hline \text { Anfipodos }} &{ } &{ } &{ } &{ } &{1} &{ } &{ } &{ } &{ } &{1} &{ } &{ } \\
{\hline \text { Sifonóforos }} &{ } &{ } &{1} &{2} &{ } &{1} &{ } &{ } &{1} &{ } &{ } &{ } \\
{\hline \text { Medusas }} &{0} &{0} &{0} &{0} &{0} &{0} &{0} &{0} &{0} &{0} &{0} &{0} \\
{\hline \text { Salpidos }} &{ } &{ } &{ } &{ } &{ } &{11} &{1} &{ } &{ } &{ } &{ } &{ } \\
{\hline \text { Apendicularias }} &{4} &{ } &{ } &{11} &{7} &{7} &{19} &{1} &{1} &{2} &{1} &{ } \\
{\hline \text { Doliolidos }} &{ } &{ } &{ } &{ } &{1} &{1} &{2} &{ } &{1} &{ } &{ } &{ } \\
{\hline \text { Eufausidos }} &{ } &{ } &{ } &{ } &{ } &{1} &{ } &{ } &{ } &{ } &{ } &{ } \\
{\hline \text { Balánidos * }} &{ } &{ } &{ } &{ } &{ } &{1} &{ } &{ } &{ } &{1} &{ } &{ } \\
{\hline \text { Bivalvos* }} &{ } &{ } &{ } &{ } &{ } &{1} &{ } &{ } &{ } &{ } &{ } &{ } \\
{\hline \text { Decápodos* }} &{10} &{3} &{4} &{1} &{6} &{6} &{2} &{2} &{ } &{3} &{3} &{2} \\
{\hline \text { Gasterópodos * }} &{ } &{ } &{ } &{ } &{ } &{ } &{2} &{ } &{ } &{ } &{ } &{ } \\
{\hline \text { Ictioplancton * }} &{1} &{ } &{ } &{ } &{1} &{ } &{ } &{2} &{1} &{1} &{ } &{ } \\
{\hline \text { Ictioplancton ** }} &{ } &{ } &{ } &{1} &{ } &{ } &{ } &{4} &{ } &{1} &{ } &{ } \\
{\hline \text { Poliquetos * }} &{ } &{ } &{1} &{ } &{ } &{1} &{ } &{1} &{ } &{ } &{ } &{ } \\
{\hline \text { Larvas Cifonautas }} &{ } &{ } &{ } &{ } &{ } &{ } &{ } &{ } &{ } &{ } &{ } &{ } \\
{\hline \text { Total }} &{135} &{9} &{41} &{110} &{117} &{170} &{210} &{63} &{15} &{98} &{32} &{8} \\
$\hline
\end{tabular}

* Larvas **Huevos 
densidades de copépodos ocurrieron en noviembre 2002 y marzo 2003, con 651 ind. $\mathrm{m}^{-3}$ y 882 ind. $\mathrm{m}^{-3}$, respectivamente, y la mínima densidad en enero 2004 (75 ind. $\mathrm{m}^{-3}$ ) (Tabla 2). Se identificó un total de 40 especies de copépodos, siendo las especies más abundantes Temora stylifera, Clausocalanus arcuicornis, Paracalanus quasimodo, P. aculeatus, Undinula vulgaris y Calanopia americana. El segundo grupo más abundante, durante el período de relajación, fue el de los apendicularias, los cuales representaron el 4,20\% del total (Tabla 2). Tuvieron sus máximas y mínimas densidades en noviembre 2002 (59 ind. $\mathrm{m}^{-3}$ ) y enero 2004 (3 ind. $\mathrm{m}^{-}$ $\left.{ }^{3}\right)$, respectivamente. Los apendicularias estuvieron representados por las especies Oikopleura longicauda, O. parva y O. rubescens. No obstante, para el período de surgencia, el segundo grupo más abundante fue el de los foraminíferos. Entre enero y marzo 2003 se observó un 'bloom' de foraminíferos en los tres estratos estudiados, representando un $10 \%$ de la abundancia de los organismos capturados en ese mes (Tabla 2). Los cladóceros, en general, no fueron muy abundantes en las muestras analizadas, hallándose con una abundancia relativamente baja (5-15\%) en marzo y septiembre 2003 y enero 2004 (Tabla 2); se identificaron las especies Pseudevadne tergestina y Penilia avirostris.

\section{Meroplancton}

Las larvas y huevos de crustáceos decápodos fueron los organismos meroplanctónicos más abundantes (correspondiendo el 2,65\% del total del zooplancton), alcanzando sus mayores abundancias en noviembre 2002 (30 ind. $\mathrm{m}^{-3}$ ), marzo y julio 2003 (20 ind. $\mathrm{m}^{-3}$ y 17 ind. $\mathrm{m}^{-}$ ${ }^{3}$, respectivamente) (Tabla 2). Las familias más comunes fueron Pinnotheridae y Xanthidae. Las larvas de balánidos (larvas cypris) ocuparon el segundo lugar en abundancia; sus picos de máxima densidad se registraron en marzo y mayo 2003, con 9 y 6 ind. $\mathrm{m}^{-3}$, respectivamente. Las larvas y huevos de peces constituyeron el $0,73 \%$ del total del zooplancton, con sus máximas densidades en noviembre de 2002 y 2003. Las larvas de Bregmaceros cantori fueron las más abundantes, seguidas de los Clupeidos y de Symphurus sp. Otro grupo presente, con muy pocos individuos, en noviembre 2002 y marzo 2003 fue el de las larvas de anélidos (Tabla 2).

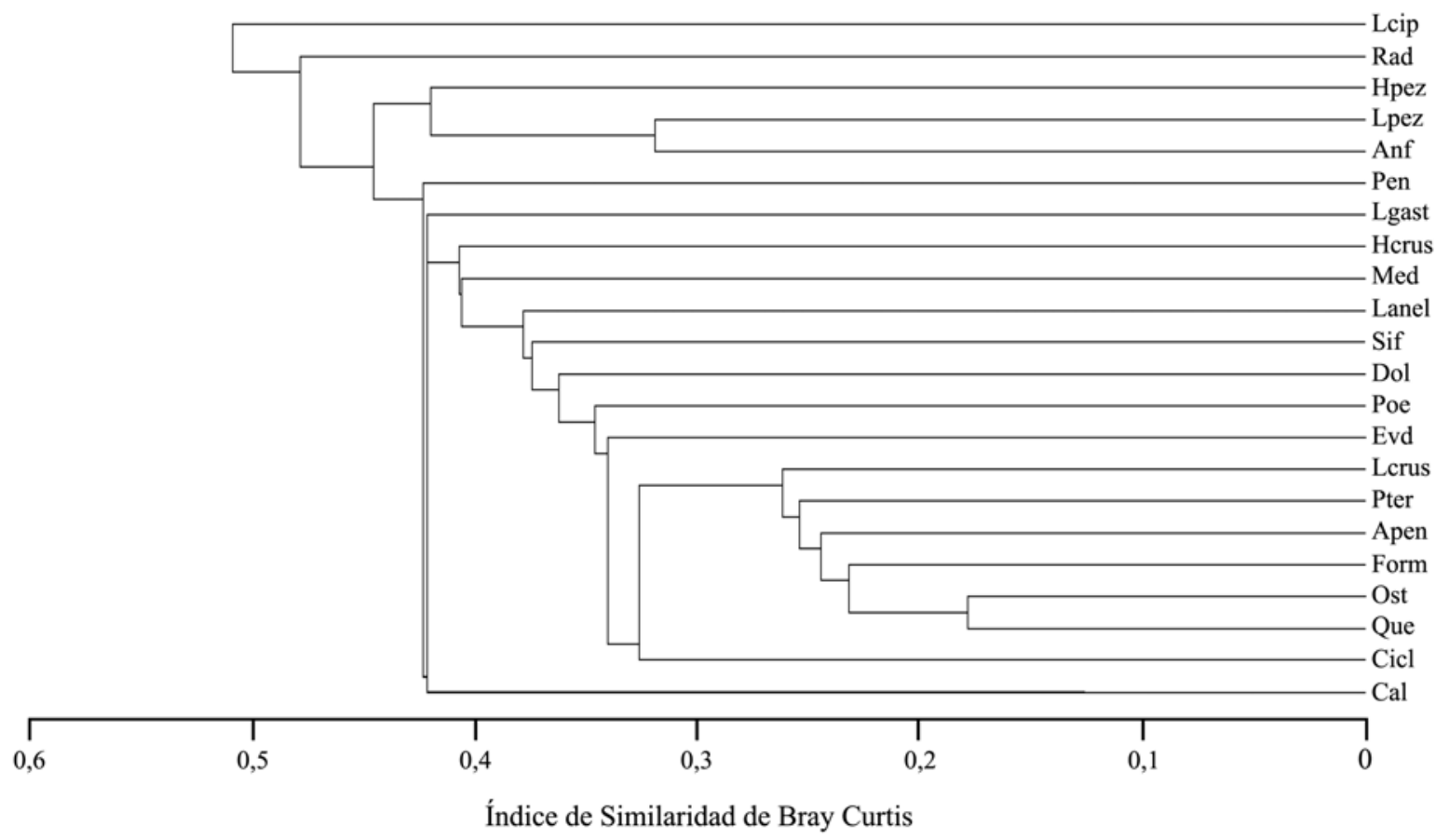

Figura 5

Dendrograma por similitud mostrando la afinidad de los organismos zooplanctónicos recolectados en la cuenca de Cariaco durante el tiempo de estudio

Cluster analysis of similarity of zooplanktonic organisms collected of the Cariaco Basin, during the study period 


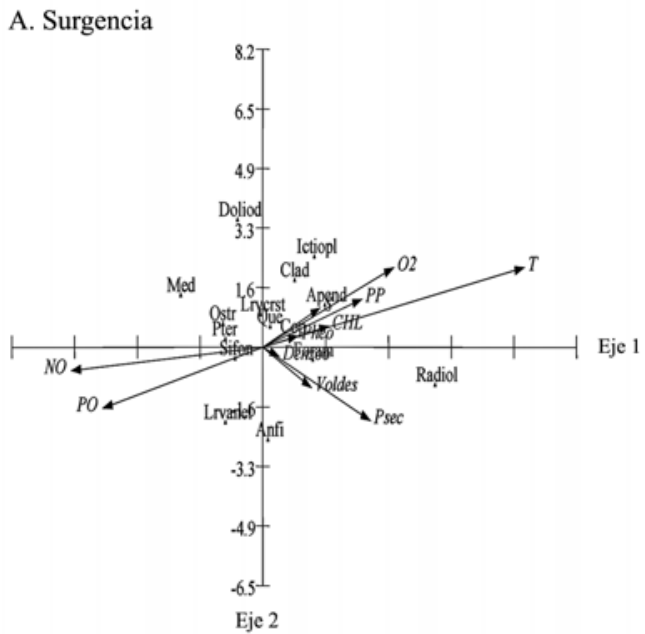

B. Relajación

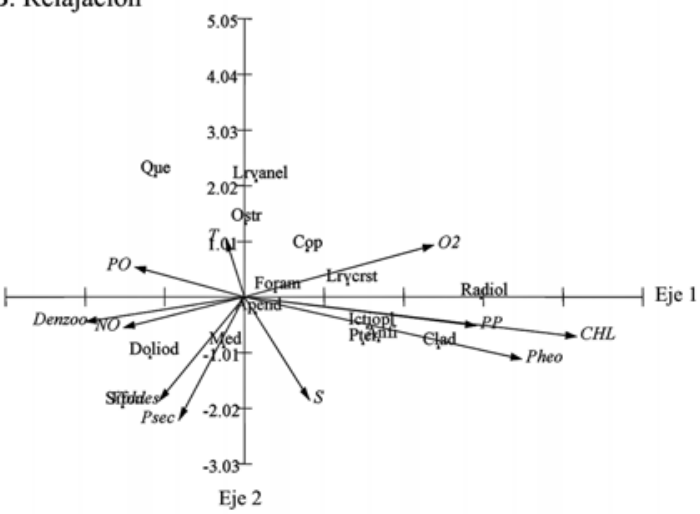

Figura 6

Análisis de correspondencia canónica $(\mathrm{ACC})$ en el período de surgencia (A) y en el período de relajación (B), donde se muestra la proyección ortogonal de los dos primeros componentes de las variables hidrográficas y los principales grupos zooplanctónicos de la cuenca de Cariaco. Pheo

(Feopigmentos), PO (Fosfato), NO (Nitrato), $\mathrm{O}_{2}$ (Oxígeno disuelto), CHL (Clorofila), T (Temperatura), Ictiopl (Ictioplancton), Lrverst (Larvas de crustáceos), Cop (Copépodos), Foram (Foraminíferos), Apend (Apendicularias), Pter (Pteropodos), Clad (Cladóceros), Sifon (Sifonóforo), Radiol (Radiolarios), Que (Quetognatos)

Canonical Correspondence Analysis (ACC) in the upwelling (A) and relaxation (B) showing orthogonal projection of the first two components of the variables basins and major zooplankton groups of the Cariaco Basin. Pheo (Pheopigments), PO (Phosphate), NO (Nitrate), $\mathrm{O}_{2}$ (dissolved oxygen), CHL (Chlorophyll), T (Temperature), Ictiopl (Ichthyoplankton), Lrvcrst (Crustacean larvae), Cop (Copepod), Foram (Foraminifera), Apend (Appendicularia), Pter (Pteropoda), Clad (Cladoceran), Sifon (Siphonophores), Radiol (Radiolarians), Que (Chaetognatha)
El análisis de clasificación ('cluster') con un corte al $40 \%$ (Fig. 5) mostró la formación de tres grupos: el primero formado por los copépodos Calanoida, Cyclopoida, Quetognatos, Ostrácodos, Foraminíferos y Apendicularias; dentro de estos grupos los más afines (70\%) son los Cyclopoida y Quetognatos, es decir grupos principalmente holoplanctónicos. El segundo grupo lo constituyen organismos holoplanctónicos y meroplanctónicos (sifonóforos, cladóceros, doliólidos, larvas de bivalvos, anélidos, crustáceos). El último grupo conformado por larvas cypris, huevos y larvas de peces, son meroplanctónico.

El análisis de correspondencia canónica (ACC) durante el período de surgencia mostró en los tres primeros componentes correlaciones positivas, con un porcentaje acumulado de $42,70 \%$ (Fig. 6A). En el primer componente se observaron claramente correlaciones positivas entre la temperatura $(\mathrm{T})$, oxígeno disuelto $\left(\mathrm{O}_{2}\right)$, biomasa zooplanctónica (peso seco y densidad), feopigmentos (Pheo) y Chl $a$ en relación inversa con los nutrientes (componentes de la surgencia costera). Los grupos del zooplancton asociados a este componente fueron los apendicularias (Apen), foraminíferos (Form), cladóceros (Clad) y copépodos (Cop), en relación inversa a los ostrácodos (Ost) y pteropodos (Pter). En el segundo componente las larvas de crustáceos (Lrvcrs) se relacionan inversamente con las larvas de anélidos (Lrvanel) y los sifonóforos (Sifon).

El ACC en el período de relajación reveló en los tres primeros componentes correlaciones positivas, con una varianza acumulada de 65,6\% (Fig. 6B). En el primer componente la relación Pheo-Chl $a$ está inversamente relacionada con la densidad zooplanctónica, junto con el nitrato (NO) y fosfato (PO); en ese mismo componente los grupos de los radiolarios (Radiol), cladóceros (Clad), ictioplancton (Ictiopl) y larvas de crustáceos (Lrvcrs) están asociados a la biomasa fitoplanctónica $(\mathrm{Chl} a)$. En el segundo componente, la temperatura (T) mostró una relación inversa a la salinidad ( $\mathrm{S}$ ) y positiva con los quetognatos (Fig. 6B).

\section{Discusión}

Durante el estudio, las aguas de surgencia constituyeron la característica hidrológica predominante de la capa superficial de la cuenca de Cariaco, como ha sido descrito por Fukuoka (1965), Okuda \& Benítez (1974) y Astor et al. (1998). En este sentido, los cambios en la abundancia, composición y distribución del zooplancton, y en especial de los copépodos pelágicos, serían evidencia del efecto de la surgencia costera, tal como lo señaló SuárezMorales (1990, 1997) para la Península de Yucatán 
durante abril de 1985. La mayor abundancia del zooplancton (enero a marzo del 2003) reflejada tanto en la abundancia como en la densidad, se corrobora por la correlación inversa con la temperatura del agua y la relación clorofila-feopigmentos. Esto sugiere que en este período la biomasa fitoplanctónica estaría controlada por el zooplancton (Burkill et al. 1987), que muestra su mayor crecimiento luego del pulso de la surgencia costera (Fraga et al. 1999).

Por otra parte, durante el período mayo-julio del mismo año, los componentes del plancton se comportaron de manera contraria. La disminución significativa de la biomasa zooplanctónica podría explicarse por la disminución en la biomasa de los productores y por el aprovechamiento del zooplancton por los niveles superiores de la trama trófica (Burkill et al. 1987). Se ha señalado que en la zona oriental de Venezuela, se produce un segundo pulso de surgencia (surgencia secundaria) entre junio y agosto (Rueda 2000, Astor et al. 2004). Las temperaturas relativamente bajas medidas en junio indicaron la ocurrencia de este fenómeno. Sin embargo, los resultados obtenidos para la biomasa planctónica (fito y zooplancton) en ese muestreo fueron bajas. Esto sugiere que el evento ocurrió muy próximo al muestreo por lo que no hubo tiempo suficiente para el crecimiento del fito y el zooplancton o que la tasa de transporte fuera del área era alta y no permitió la acumulación de los organismos. Los resultados de este estudio reflejan una amplia variabilidad temporal en la composición y abundancia de la comunidad zooplanctónica, a pesar de que la estación está ubicada en un área tropical.

La detección de un tercer pico de biomasa y densidad zooplanctónica en el estrato profundo en noviembre de 2003 mostró el hundimiento de la capa de mayor producción de zooplancton, que coincide con el cambio de las condiciones ambientales, es decir, una mayor estabilidad de la columna de agua (septiembrenoviembre), una menor concentración de nutrientes y de Chl $a$, presentándose alta estratificación. Por ello, posiblemente afecta la ubicación de la nutriclina hacia zonas más profundas. Este proceso, lo detectó previamente Rueda (2000) ubicando los máximos de clorofila $a$, alrededor de $\operatorname{los} 50 \mathrm{~m}$, unido a una elevada concentración de fitoplancton subsuperficial. Igualmente Astor et al. (2004) indicaron que entre agosto y noviembre se encuentran las poblaciones de fitoplancton más longevas, diversas y eficientes (inferido del índice de pigmentos), como consecuencia de la estratificación de las aguas y la escasez de nutrientes. Los niveles de producción primaria, aunque bajos, continúan en la superficie con características uniformes en un intervalo mayor de profundidad (alrededor de $1 \mathrm{mgC} \mathrm{m}^{-2} \mathrm{~h}^{-1}$ hasta los $55 \mathrm{~m}$ ). Por lo tanto, el ciclo se reinicia de nuevo a finales de año.

Aunque se detectaron cambios temporales, la biomasa, densidad y composición del zooplancton por estrato no mostró diferencias significativas, ya que en el período de surgencia los máximos valores se registraron en la capa superficial, mientras que en relajación se detectaron en la capa profunda. Esto es producto de que la variabilidad anual de ambas capas es similar.

A pesar de su gran profundidad, el área de estudio está ubicada cerca a la plataforma continental, y por consiguiente, los valores obtenidos de producción secundaria son comparables a los detectados en otras zonas costeras tropicales (Marikova \& Campos 1967, Kolesnikov \& Alfonso 1969). No obstante, los valores fueron ligeramente mayores que los reportados por Peñuela (2000) para la cuenca de Cariaco (25,85-579,59 $\mathrm{mg} \mathrm{m}^{-3}$ de peso seco). Sin embargo, al comparar los valores de la biomasa zooplanctónica con los obtenidos en la presente investigación se nota que las estimaciones son mucho más bajas que las reportadas para la misma zona. Esta diferencia en la productividad secundaria podría ser atribuida al dinamismo que presentan las comunidades planctónicas y en las cuales no se puede esperar que se repitan valores similares, ya que existen variaciones en los ciclos biológicos de las poblaciones y fluctuaciones interanuales en la climatología que pueden intervenir para que la biomasa sea alta o baja en un espacio dado y en un momento determinado (Sameoto 1984, Morales \& Murillo 1996).

El zooplancton puede alterar las concentraciones de C en el océano a través de la respiración, consumo del fitoplancton, excreción de compuestos orgánicos, y por migración diaria de las capas superficiales donde se alimenta, a las capas más profundas (Astor et al. 2004). En consecuencia, el zooplancton es un componente que acelera la bomba biológica, influyendo en la pérdida de materia orgánica de las capas superficiales a las aguas profundas donde puede salir del sistema pelágico (Astor et al. 2004).

La composición del zooplancton en la cuenca de Cariaco estuvo dominada por copépodos, los cuales constituyen aproximadamente entre el $50 \%$ y el $80 \%$ del total del zooplancton, tanto en aguas oceánicas como en zonas neríticas y costeras (Boltovskoy 1981, Alder \& Boltovskoy 1993, Gasca \& Suárez 1996). En este estudio los copépodos dominaron más del $60 \%$ de la abundancia total del zooplancton, tanto para el período de relajación como el de surgencia. Resultados similares fueron obtenidos por Zoppi (1977), Urosa $(1977,1983)$ y Peñuela (2000) para el golfo y cuenca de Cariaco. En 
otras zonas del mundo, autores como Rodríguez \& Rubín (1991), Alder \& Boltovskoy (1993), Webber et al. (1996), Hulsemann (1996) y Fernández-Puelles et al. (1997) confirman el dominio de este grupo en las comunidades zooplanctónicas.

La estructura de la comunidad de copépodos mostró ser una consecuencia de la condición local de la surgencia, con altas densidades de especies herbívoras en el área, donde las concentraciones de alimento ( $\mathrm{Chl} a$ ) con la presencia de diatomeas coloniales como Chaetoceros spp., Climacodium frauenfeldianum, Guinardia striata, Hemiaulus sinensis y diatomeas pennadas como Meuniera membranacea, Pseudonitzschia sp. y Thalassionema nitzschioides (Díaz-Ramos com. pers.) son altas, lo que evidencia la actividad de pastoreo por parte del zooplancton.

Algunos autores han relacionado los copépodos pelágicos con surgencia. Margalef (1971), SuárezMorales (1990, 1997) mostraron que los elementos dominantes del zooplancton en sistemas de surgencia son principalmente copépodos herbívoros de pequeño tamaño, tales como Oithona, Paracalanus, Eucalanus y Clausocalanus. En el área estudiada, los géneros de copépodos pequeños, Temora, Paracalanus y Eucalanus fueron encontrados claramente abundantes con el agua de surgencia.

En los resultados obtenidos, las especies más abundantes y dominantes durante el período de surgencia fueron Temora stylifera, Clausocalanus arcuicornis, Paracalanus quasimodo, P. aculeatus, Undinula vulgaris y Calanopia americana, las cuales son especies comunes $\mathrm{y}$ frecuentes en aguas costeras ricas en nutrientes en el Mar Caribe y aguas adyacentes (Bjornberg 1981; SuárezMorales 1990, 1997).

Algunos autores como Bjornberg (1981) y Gasca \& Suárez (1996) afirman que especies como Temora stylifera, Paracalanus aculeatus, Calocalanus pavo y Clausocalanus furcatus presentan afinidad con las aguas cálidas tropicales típicas de la región. Sin embargo, las especies T. stylifera, C. pavo, C. furcatus y Euchaeta marina fueron caracterizadas por Owre \& Foyo (1964) como formas oceánicas atlánticas, la presencia de estas especies en la cuenca podrían explicarse por el fenómeno de surgencia, así como por la libre comunicación que tienen las aguas de la cuenca con las aguas vecinas del océano Atlántico, las cuales podrían ser transportadas hacia aguas del mar Caribe, por el efecto de las diversas corrientes o remolinos (Astor et al. 2003, 2004). En ese sentido, en el Mar Caribe se ha observado la presencia de remolinos fluyendo hacia el noroeste con la corriente del Caribe (Pauluhn \& Chao 1999). En ocasiones, estos remolinos circulan en áreas cercanas de la plataforma continental venezolana siendo su origen variable. Algunos giros se generan fuera del Mar Caribe, al desprenderse de la zona de retroflexión de la corriente norte de Brasil, y otros se desarrollan a partir de la corriente del Caribe. Este fenómeno induce la intrusión de aguas del Caribe a la cuenca sobre el umbral del canal de La Tortuga (Astor et al. 2003, 2004); por lo tanto, podrían identificarse especies de copépodos características de aguas oceánicas del Atlántico. En este sentido, se identificaron las especies Euchaeta tonsa y E. spinosa, las cuales han sido consideradas como especies de aguas profundas del Atlántico norte occidental (Owre \& Foyo 1964; Bjornberg 1981, Suarez-Morales 1990).

La distribución vertical de los copépodos mostró que son en efecto bioindicadores del proceso de surgencia costero típico de la región (Fukuoka 1965, Okuda \& Benítez 1974), puesto que aparecen sus mayores concentraciones desde noviembre de 2002 hasta marzo 2003. En estos meses, se evidenció claramente el proceso de mezcla en toda la columna de agua $(<100 \mathrm{~m})$, ya que se obtuvieron altas concentraciones de este grupo en los tres estratos estudiados. Esto coincide con los resultados obtenidos por Cervigón \& Marcano (1965) quienes encontraron un máximo de copépodos en febrero y marzo en la cuenca de Cariaco a nivel superficial $(<100 \mathrm{~m})$.

El zooplancton estuvo compuesto mayormente por holoplancton (copépodos, cladóceros y apendicularias; Tabla 2) lo cual es característico de aguas oceánicas (Zoppi 1977, Rodríguez \& Rubin 1991, Morales y Murillo 1996, Castellanos y Suárez-Morales 1997). En áreas oceánicas, este grupo de organismos es más diverso que el meroplancton (Reeve 1975, Margalef 1971). Resultados similares fueron obtenidos por Morales \& Murillo (1996) y Biancalana et al. (2007) quienes detectaron diferencias significativas en la abundancia y diversidad del holoplancton y el meroplancton. En esos estudios, las poblaciones del primero dominaron tanto en número como en diversidad, sugiriendo que la baja diversidad de las formas larvales es producto de la fuerte sedimentación y resuspensión.

Los grupos meroplanctónicos presentes en las muestras (larvas de crustáceos, moluscos y el ictioplancton) evidencian el transporte horizontal mar afuera de las corrientes marinas locales y la surgencia. Estos grupos, junto con los ítems netamente neríticos, podrían utilizarse como indicadores de la surgencia en la cuenca (Marín et al. 1989).

La variabilidad temporal del zooplancton sugiere que, la producción secundaría del área de la cuenca de Cariaco no es constante durante todo el año, pudiéndose constatar 
igualmente con los resultados obtenidos en la densidad de organismos y biomasa, lo cual dependen en gran parte de los factores físicos que inducen la productividad fitoplanctónica. Asimismo, el análisis cualitativo sugiere que el zooplancton de la cuenca de Cariaco corresponde más a un zooplancton pelágico-oceánico que a un zooplancton demersal. Las larvas de crustáceos, larvas de moluscos y poliquetos, ictioplancton, así como los copépodos, apendicularias y quetognatos son más abundantes en muestras no demersales (Ferraris 1982). Sin embargo, la alta variabilidad en sus distribuciones es una consecuencia directa de los cambios relativamente rápidos en el tiempo y el espacio de la comunidad zooplanctónica (Greenblatt 1982).

\section{Agradecimientos}

Este trabajo ha sido financiado por la National Science Foundation (NSF subvención OCE-9729284 y OCE9216626 otorgadas a Frank Müller-Karger y subvenciones OCE-9415790, OCE- 9711318, y OCE9730278 otorgadas a Mary Scranton y Gordon Taylor, y por el Consejo Nacional de Investigaciones Científicas y Tecnológicas (CONICIT, VENEZUELA, subvención 96280221). Estamos agradecidos al Dr. Pablo Mandazen (Hermano Ginés, Director FLASA) por su apoyo y a Yrene Astor por su liderazgo y empeño en llevar a cabo los cruceros y también a la tripulación del B/O Hermano Ginés (FLASA). También agradecemos a los evaluadores del manuscrito por sus pertinentes sugerencias.

\section{Literatura citada}

Alder V \& D Boltovskoy. 1993. The ecology of larger microzooplankton in the Weddell Scotia confluence area: horizontal and vertical distribution patters. Journal of Marine Research 51: 323-344.

Angel MV. 1998. Pelagic biodiversity. En: Ormond RFG, JD Gage, MV Angel (eds). Marine biodiversity: patterns and processes, pp. 35-68. Cambridge University Press, Cambridge.

Astor Y, M Jenifer \& F Müller-Karger. 1998. Variabilidad estacional hidrográfica en la Cuenca de Cariaco. Memorias de la Sociedad Natural La Salle 149: 61-72.

Astor Y, F Müller-Karger \& M Scranton. 2003. Seasonal and interannual variation in the hydrography of the Cariaco Basin: Implication for basin ventilation. Continental Shelf Research 23(1): 125-144.

Astor Y, F Müller-Karger, R Bohrer, L Troccoli \& J García. 2004. Variabilidad estacional e interanual del carbono inorgánico disuelto y nutrientes en la Cuenca de Cariaco. Memorias de la Sociedad Natural La Salle 161-162: 235 252.

Berasategui A. 2003. An historical review of the planktonic copepods diversity of the Río de la Plata and the Argentine-
Uruguay common fishing zone. PNUD Project/Gef RLA/ 99/G31. Argentina. Informe Técnico, Proyecto FREPLATA, 22 pp. [en línea] <http://www.freplata.org/documentos/ archivos/Documentos_Freplata/Planktonic_Biodiv.pdf >

Biancalana F, MS Barría de Cao \& MS Hoffmeyer. 2007. Micro and mesozooplankton composition during winter in Ushuaia and Golondrina Bays (Beagle Channel, Argentina). Brazilian Journal of Oceanography 55(2): 83-95.

Bjornberg TK. 1981. Copepoda. En: Boltovskoy D (ed). Atlas del zooplancton del Atlántico Sudoccidental y métodos de trabajo con el zooplancton marino, pp. 587-679. Publicación Especial del INIDEP, Mar de Plata.

Boltovskoy D. 1981. Atlas del zooplancton del Atlántico Sudoccidental y métodos de trabajo con el zooplancton marino, 936 pp. Publicación Especial del INIDEP, Mar de Plata.

Boltovskoy D (ed). 1999. South Atlantic zooplankton. Vol. 12: 1-1706. Backhuys Publishers, Leiden.

Boyer J, J Fourqurean \& R Jones. 1997. Spatial characterization of water quality in Florida Bay and whitewater bay by multivariate analyses: zones of similar influence. Estuaries 20(4): 743-758.

Burkill P, R Mantoura, C Llewellyn \& N Owens. 1987. Microzooplankton grazing and selectivity of phytoplankton in coastal waters. Marine Biology 93: 581-590.

Castellanos I \& E Suárez-Morales. 1997. Observaciones sobre el zooplancton de la zona arrecifal de Mahahual, Quintana Roo (Mar Caribe). Anales del Instituto de Biología, Universidad Nacional Autónoma de México, Serie Zoología 68(2): 237-252.

Cervigón F \& P Marcano. 1965. Zooplancton. Estudios sobre el ecosistema pelágico del NE de Venezuela. Memorias de la Sociedad Natural La Salle 70: 363-268.

Febres G. 1974. Circulación de las aguas superiores de la Cuenca de Cariaco. Boletín del Instituto Oceanográfico de Venezuela, Universidad de Oriente 13(1-2): 79-90.

Fernández-Puelles M, I Jansá, C Gomis, D Gras \& B Amengual. 1997. Variación anual de las principales variables oceanográficas y planctónicas en una estación nerítica del Mar Balear. Boletín del Instituto Español de Oceanografía 13(1/2): 13-33.

Ferraris JD. 1982. Surface zooplankton at Carrie Bow Cay, Belize. Smithsonian Contributions Marine Science 12: 239251.

Fraga F, A Ríos, F Pérez, M Estrada, \& C Marrasé. 1999. Effect of upwelling pulses on excess carbohydrate synthesis as deduced from nutrient, Carbon dioxide and oxygen profiles. Marine Ecology Progress Series 189: 65-75.

Fukuoka J. 1965. Coastal upwelling near Venezuela. (I). Year to year change of upwelling. Boletín del Instituto Oceanográfico de Venezuela 4(2): 223-233.

Gasca R \& E Suárez. 1996. Introducción al estudio del zooplancton marino, 711 pp. El colegio de la Frontera Sur (ECOSUR), Chetumal.

Gómez A. 1996. Causas de la fertilidad en el Nororiente de 
Venezuela. Interciencia 21(3): 140-146.

Gordon L, Jr Jennings, A Ross \& J Crest. 1993. A suggested protocol for continuous flow automated analysis of seawater nutrients. In: WOCE Operation Manual. WHP Office Report 90-1, WOCE Report 77 (68/91): 1-52.

Greenblatt PR. 1982. Small-scale horizontal distribution of zooplankton taxa. Marine Biology 67: 97-111.

Hulsemann K. 1996. Copepoda. En: Gasca R \& E Suárez (eds). Introducción al estudio del zooplancton marino, pp. 250294. El Colegio de la Frontera Sur (ECOSUR), Chetumal.

Johnson R \& D Wichern. 1992. Applied multivariate statistical analysis, 642 pp. Prentice-Hall, London.

Kolesnikov AN \& A Alfonso. 1969. Datos preliminares del zooplancton de la región oriental del Golfo de México y el estrecho de Florida. Academia de Ciencias de Cuba. Serie Oceanologica 4: 15-20.

Legaré JE. 1961. Estudios preliminares del zooplancton en la región de Cariaco. Boletín del Instituto Oceanográfico de Venezuela 1(1): 191-218.

Legendre L \& P Legendre. 1998. Numerical ecology, 418 pp. Elsevier, Amsterdam.

Margalef R. 1971. The pelagic ecosystem of the Caribbean Sea. Symposium on Investigations and resources of the Caribbean Sea and Adjacent Regions, UNESCO, Paris, pp. 345-367.

Marikova VK \& A Campos. 1967. Características cualitativas y cuantitativas del zooplancton de la plataforma cubana. Academia de Ciencias de Cuba, Instituto de Oceanología 2(2): $63-80$

Marín B, A Gómez \& L Urosa. 1989. Taxonomía y distribución del ictioplancton en la Cuenca de Cariaco. Boletín del Instituto Oceanográfico de Venezuela 28(1/2): 1528.

Martin JW \& GE Davis. 2001. An updated classification of the recent Crustacea. Natural History Museum of Los Angeles County, California, Science Series 39: 1-123.

McEven GF, MW Johnson \& TR Folsom. 1954. A statistical analysis of the performance of the Folsom Plankton Splitter based upon test observations. Archiv fur Meteorologie, Geophysiks und Bioklimatologie. Serie A, Meteorologie und Geophysik 1(17): 167-175.

Morales A \& M Murillo. 1996. Distribution, abundance and composition of coral reef zooplankton, Cahuita National Park, Limon, Costa Rica. Revista de Biología Tropical 44(2): 619-630.

Müller-Karger F, C Hu \& R Varela. 1999. Validation of carbon flux and related products for SIMBIOS: the CARIACO continental margin time series and the Orinoco river plume. En: McClain CR \& GS Fargion (eds). SIMBIOS Project 1999 Annual Report, pp. 88-92. NASA, Goddard Space Flight Center, Greenbelt. [en línea] <http:/ /seabass.gsfc.nasa.gov/docs/SIMBIOS_Annual_1999.pdf>

Müller-Karger F, R Varela, R Thunell, M Scranton, R Bohrer, G Taylor, J Capelo, Y Astor, E Tappa, TY Ho \& JJ Walsh. 2001. Annual cycle of primary production in the Cariaco Basin: Response to upwelling and implications for vertical export. Journal of Geophysical Research 106(C3): 4527-4542.

Okuda T \& J Benítez. 1974. Condiciones hidrográficas de las capas superiores en la Cuenca de Cariaco y áreas adyacentes durante la época lluviosa. Boletín del Instituto Oceanográfico de Venezuela 13(1/2): 147-162.

Okuda T, B Gamboa \& A Yegres. 1969. Seasonal variations of hydrographic conditions in the Cariaco Trench. Boletín del Instituto Oceanográfico de Venezuela 8(1/2): 21-27.

Owre H \& M Foyo. 1964. Report on a collection of Copepoda from the Caribbean Sea. Bulletin of Marine Science 14(2): 359-372.

Parsons T, Y Maita \& C Lally. 1984. A manual of chemical and biological methods of seawater analysis, $173 \mathrm{pp}$. Pergamon Press, Oxford.

Pauluhn A \& Y Chao. 1999. Tracking eddies in the subtropical north-western Atlantic Ocean. Physical Chemistry. Earth A24(4): 415-421.

Peñuela M. 2000. Análisis cuantitativo y cualitativo del zooplancton superficial de la Cuenca de Cariaco, Venezuela. Tesis de Biología, Departamento de Biología, Escuela de Ciencias, Universidad de Oriente, Cumaná, 100 pp.

Postel L, H Fock \& W Hagen. 2000. Biomass and abundance. En: Harris RP, PH Wiebe, J Lenz, HR Skjoldal \& M Huntley (eds). ICES zooplankton methodology manual, pp. 83-174. Academic Press, San Diego.

Reeve MR. 1975. The ecological significance of the zooplankton in the shallow subtropical waters of South Florida. En: Cronin LE (ed). Estuarine Research. 1. Chemistry, biology, and the estuarine system, pp. 352-371. Academic Press, New York.

Richards F. 1960. Some chemical and hydrographic observations along the north coast of South America 1. Cabo Tres Puntas to Curacao, including the Cariaco Trench and the Gulf of Cariaco. Deep Sea Research 7: 163-182.

Richards F \& RF Vaccaro. 1956. The Cariaco Trench, an anaerobic basin in the Caribbean Sea. Deep-Sea Research 3(3): 214-228.

Rodríguez J \& J Rubín. 1991. El ictioplancton y la biomasa del zooplancton en aguas del sur de Galicia, en abril 1987. Boletín del Instituto Español de Oceanografía 7(2): 115127.

Rueda D. 2000. Variabilidad temporal de la distribución vertical de la biomasa fitoplanctónica en la depresión oriental de la cuenca de Cariaco y sus relaciones con los aspectos hidrográficos del estrato superficial (1996-1998). Tesis Magister Scientiarum en Biología Marina, Universidad de Oriente, Cumana, 120 pp.

Sameoto DD. 1984. Environmental factors influencing diurnal distribution of zooplankton and icthyoplankton. Journal of Plankton Research 6: 767-792.

Scranton MI, Y Astor, R Bohrer, TY Ho \& F Müller-Karger. 2001. Controls on temporal variability of the geochemistry of the deep Cariaco Basin. Deep-Sea Research 1(48): 16051625 . 
Siegel S \& NJ Castellan. 1995. Estadística no paramétrica aplicada a la conducta, $437 \mathrm{pp}$. Trillas, México.

Smith DL. 1977. A guide to marine coastal plankton and invertebrate larvae, $161 \mathrm{pp}$. Kendall/Hunt, Dubuque.

Strickland RD \& TR Parsons. 1972. Manual of sea water analysis. Bulletin of the Fisheries Research Board of Canada 125: $1-310$.

Suárez-Morales E. 1990. Planktonic copepods: A note on their relation with upwelling areas in the Campeche bank and the Mexican Caribbean Sea. Investigaciones Marinas CICIMAR 5(1): 87-92.

Suárez-Morales E. 1997. Pelagic copepod assemblages during spring upwelling off the Yucatan Peninsula (1985). Intergovernmental Oceanographic Commission (SCOR/ UNESCO), Workshop Report 142: 345-352.

Trégoubooff G \& M Rose. 1957. Manuel de planctonologie Méditerranéenne 2: 1-199. Centre National de la Recherche Scientifique, Paris.

Urosa L. 1977. Bibliografía y observaciones sobre el zooplancton del mar Caribe. Lagena 39/40: 11-26.

Urosa L. 1983. Distribución del zooplancton en la cuenca TuyCariaco, área de posible actividad petrolera en Venezuela. Boletín del Instituto Oceanográfico de Venezuela 22(1/2): 125-143.
Van Geen A, D McCorkle \& G Klinkhammer. 1995. Sensitivity of the phosphate-cadmium-carbon isotope relation in the ocean to cadmium removal by suboxic sediments. Paleoceanography 10(2): 159-169.

Walsh JJ, DA Dieterle, F Müller-Karger, R Bohrer, WP Bissett, RJ Varela, R Aparicio, R Díaz, R Thunell, GT Taylor, MI Scranton, KA Fanning \& ET Peltzer. 1999. Simulation of carbon/nitrogen cycling during spring upwelling in the Cariaco Basin. Journal of Geophysical Research 104(C4): 7807-7825.

Webber M, J Roff, L Chisholm \& C Cheryl. 1996. Zooplankton distributions and community structure in an area of the South Coast Shelf of Jamaica. Bulletin of Marine Science 59(2): 259-270.

Zoppi E. 1961. Distribución vertical del zooplancton en el Golfo y extremo este de la Cuenca de Cariaco. Boletín del Instituto Oceanográfico de Venezuela, Universidad de Oriente 1(1): 219-248.

Zoppi E. 1977. El zooplancton marino de la región oriental de Venezuela. Trabajo de Postgrado, Doctorado en Ciencias, Universidad Central de Venezuela, Caracas, 187 pp.

Recibido el 12 de febrero de 2009 y aceptado el 28 de septiembre de 2009 